$\mathcal{B} \mathcal{T P} \mathcal{S}$

Brazilian Transportation

Planning Society
Journal of Transport Literature

Vol. 6, n. 4, pp. 215-227, Oct 2012

Reviews \& Essays
JTL | RELIT

www.transport-literature.org ISSN 2238-1031

\title{
Fusões no transporte aéreo: estudos e tendências
}

[Mergers in air transportation: studies and trends]

Thiago Vinicius Alves Ueda*

Instituto Tecnológico de Aeronáutica (ITA), Brazil

Submitted 20 Sep 2011; received in revised form 18 Dec 2011; accepted 26 Jan 2012

\begin{abstract}
Resumo
0 presente artigo apresenta recentes estudos da literatura acerca dos efeitos das fusões para o bem-estar social dos usuários do transporte aéreo, mais especificamente, como o processo impacta no preço das passagens e nas frequências de voos. Os resultados sugerem que fusões entre companhias aéreas "legacy" nos EUA tem causado significante aumento nos preços das passagens e redução nas frequências de voo. Além disso, fusões parecem resultar em redução de preços quando companhias low. cost estão envolvidas, salvo bilhetes adquiridos com poucos dias de antecedência. Por fim, fusões entre companhias network e low-cost mostram-se prejudiciais para os consumidores, mas a magnitude do impacto varia de acordo com a competição entre as companhias anteriormente em rotas específicas.
\end{abstract}

Palavras-Chave: fusões; transporte aéreo; preço; frequência; bem-estar social.

\section{Abstract}

This article presents new studies of the literature about the effects of mergers to the social welfare of the users of air transport, more specifically, as the process impacts the price of the tickets and on the frequency of flights. The results suggest that mergers between legacy carriers in the USA has caused significant increases in the prices of the tickets and reduction in the flight frequency. In addition, mergers seem to result in reduction of prices when low-cost companies are involved, except for tickets purchased with few days in advance. Finally, mergers between network carriers and low-cost carriers are non-benefit to consumers, however the impact depends on the competition between the carriers previously in specific routes.

Key words: mergers; air transportation; ticket prices; flight frequencies; social welfare.

* Corresponding Author. Email: uedathiago@gmail.com.

\section{Recommended Citation}

Ueda, T. V. A. (2012) Fusões no transporte aéreo: estudos e tendências. Journal of Transport Literature, vol. 6, n. 4, pp. 215 -227.

- JTL/RELIT is a fully electronic, peer-reviewed, open access, international journal focused on emerging transport markets and published by BPTS - Brazilian Transport Planning Society. Website www.transport-literature.org. ISSN 2238-1031.

This paper is downloadable at www.transport-literature.org/open-access. 


\section{Introdução}

O presente artigo apresenta recentes estudos da literatura acerca dos efeitos das fusões para o bem-estar social dos usuários do transporte aéreo, mais especificamente, como o processo impacta no preço das passagens e nas frequências de voos. Em síntese, literatura evidencia que fusões entre companhias aéreas "full service" no mercado norte americano tem causado significante aumento nos preços das passagens e redução nas frequências de voo, e pouca coisa pode-se afirmar sobre o impacto em preços pós-fusão em outros países. Além disso, fusões parecem resultar em redução de preços quando companhias low-cost estão envolvidas, exceto para tickets adquiridos com poucos dias de antecedência. Além disso, fusões parecem resultar em redução de preços quando companhias low-cost estão envolvidas, salvo bilhetes adquiridos com poucos dias de antecedência. Por fim, fusões entre companhias network e low-cost mostram-se prejudiciais para os consumidores, mas a magnitude do impacto varia de acordo com a competição entre as companhias anteriormente em rotas específicas.

O transporte aéreo passou por um intenso processo de liberalização nas últimas décadas, como um legado, tem-se notado um aumento exacerbante do número de companhias aéreas operando em todo o mundo. Neste contexto, uma das preocupações do período atual tem sido a tendência do aumento do número de fusões e acordos codeshare entre companhias aéreas. Se por um lado, esta mudança organizacional pode garantir a sobrevivência das empresas operantes num mercado complexo, e servir como barreira às novas entrantes, por outro lado, fusões podem ser prejudiciais ao bem estar dos passageiros ao diminuir, em certos casos, a concorrência nos mercados e acarretar em tarifas onerosas e diminuição do número de frequências.

Os impactos da recessão global e os ataques terroristas de 11 de setembro provocaram uma reestruturação tardia da indústria da aviação. Em qualquer outro setor, a consolidação teria ocorrido, naturalmente, com relativa antecedência. Atualmente, considerando que muitas firmas lutam para sustentar sua viabilidade financeira, há uma janela de oportunidade para redesenhar o modelo de negócios da aviação. O resultado tem sido uma onda de fusões que está resultando em uma indústria da aviação mais competitiva e eficiente em termos globais, com um número mais limitado de empresas, tanto nos EUA como na Europa, sendo capaz de 
servir um conjunto mais amplo de necessidades dos usuários.

Independente do setor, mais de 50 por cento de todas as fusões ficam aquém de seus objetivos declarados Wood et al (2010). Fusões de companhias aéreas não são exceções, na verdade, elas têm produzido alguns fracassos consideráveis. Parte das companhias aéreas está auferindo perdas dramáticas, reduções de capacidade, e cortes de pessoal. Por exemplo, a renomada Swissair entrou em processo de falência e a liquidação está em andamento. Neste contexto, a consolidação parece ser a principal saída para o dilema estrutural da indústria.

A própria estrutura do mercado da aviação é capaz de explicar as dificuldades no período pósfusão: choques cíclicos de demanda, instabilidade do mercado petrolífero e ainda, coação política e religiosa manifestada através de terrorismo. Além disso, outro possível fator que explica o insucesso nos processos de Fusões e aquisições é o grau de aplicação de abordagens reducionistas deturpadas. Wood et al (2010) pontuam que: "Muitos executivos e consultores desenvolvem modelos conceituais muito simples, que são usados para avaliar questões organizacionais complexas. Por trás dos maus resultados de muitos processos de fusão e aquisição encontra-se uma abordagem reducionista que não reconhece a complexidade $e$ todas as dimensões envolvidas". Alternativamente a este tipo de abordagem, têm se o pensamento sistêmico que contempla as diversas dimensões envolvidas no processo de combinação de diferentes empresas para a criação de uma nova sociedade. Fusões são processos complexos, cujas condições para o sucesso ainda não são totalmente inteligíveis, entretanto, adotar uma perspectiva sistêmica, sensível a fatores externos, hábil na condução do processo, contar com certa dose de sorte são fatores impreteríveis para dar início a esta forma de mudança organizacional.

O presente trabalho está assim dividido: a Seção 2 introduz conceitos básicos sobre o processo de fusões, a Seção 3 apresenta um breve histórico de fusões no transporte aéreo, a Seção 4 apresenta estudos recentes da literatura acerca do processo de fusões. E por fim, nas Conclusões, denotou-se que as diferenças no impacto da fusão são substanciais, dependendo do tipo de companhia aérea envolvidas, mas também da localização geográfica das rotas e do perfil do passageiro. Assim, concluímos que qualquer avaliação de uma fusão precisa para levar em conta a percepção da diferenciação dos produtos de uma "network carrier" e uma low-cost. 


\section{Conceitos sobre Fusões}

A Lei 6404/76 brasileira define fusão como o processo que levaria ao desaparecimento das firmas envolvidas, resultando numa nova empresa. Entretanto na prática, o ambiente corporativo, e até a academia, tem usado, erroneamente, os termos cisão, incorporação, aquisição e fusão intercambiavelmente. Além disso, com frequência, tem-se usado o termo fusão acompanhado do termo aquisição, isto explica a abreviatura "F\&A". Alheio a estas divergências conceituais e terminológicas, usaremos a definição legislativa neste artigo, sem perda de generalidade.

Há diversos fatores que tentam explicar as razões pelo qual os processos de fusão e aquisição ocorrem. Intuitivamente, o processo de "F\&A" deve-se majoritariamente a motivos estratégicos e ou financeiros. Entretanto, não nos convém discutir em pormenores estas possíveis razões, interessa-nos apresentar os possíveis e potenciais ganhos ao se incorrer neste processo de mudança organizacional. Sociedades fusionadas obtêm mais vantagens como:

- Forma alternativa de Investimento

- Acesso facilitado ao mercado de capitais, onde os recursos são mais estáveis e as taxas de juros mais baixas.

- Diversificação do portfólio de produtos

- Diminuição inerente do risco global ao qual a empresa fica exposta.

- Acesso rápido a mercados outrora inexplorados.

- Sinergia: economias de escala, escopo e densidade

- Eliminações de funções redundantes

- Otimização das operações de logística.

- Aumento da participação de mercado.

- Interesses pessoais dos executivos

No transporte aéreo, uma das maneiras mais eficiente de alcançar maior participação de mercado e afastar concorrentes é através de fusões e aquisições. Não é a única forma de atingir este objetivo, tem-se empregado os acordos codeshare e acordo com os aeroportos para ter poder de veto a investimentos ou mesmo a compra de terminais, por exemplo. Vale ressaltar que este último cenário aplica-se a realidade internacional. 


\section{Histórico de fusões no transporte aéreo}

A primeira onda de fusões aconteceu na década de 60 no mercado estadunidense, e a partir do início deste século um número considerável de fusões aconteceu em outros continentes. Exemplos de fusões recentes que ocorreram na Europa e EUA podem ser encontrados entre companhias aéreas "network": Air France e KLM, British Airways e Iberia, Delta e Northwestern, Lufthansa e Swiss, Austrian e BMI, United e Continental, US Airways e American West. Entre companhias aéreas "low-cost": Air Berlin e LTU e Condor, Easyjet e Go Fly, Ryanair e Buzz, Southwest e AirTran. Além disso, na Europa, uma prática comum é que companhias aéreas low-cost tornem-se subsidiárias de companhias aéreas "network", podemos citar: Germanwings e bmibaby da Lufthansa, Transavia da Air France-KLM, Vueling da Iberia e a maior companhia low-cost da Europa, Ryanair, tem tentado fusionar com a companhia aérea "network", Aer Lingus.

A tabela 1 pormenoriza o histórico de fusões, fornecendo dados sobre a data do acordo, as companhias aéreas envolvidas e o tipo de mudança organizacional: fusão, aquisição, etc. É notável, que os processos de fusão são predominantes no mercado de aviação comercial de passageiros. Poucas empresas de carga optaram por fusionar sociedades, conforme indica a Tabela 2. Atemo-nos ao mercado dos EUA por ser o mais relevante.

Tabela 1 - Histórico de fusões de companhias aéreas de transporte de passageiros. Fonte: Artigo "Merger Mania”, Elaboração própria.

\begin{tabular}{lll}
\hline Companhia & Ano & Disposição \\
\hline Capital & 1961 & fusão com United \\
AAXICO & 1965 & fusão com Saturn \\
Mackey & 1967 & fusão com Eastern \\
PANAGRA & 1967 & fusão com Braniff \\
Pacific Northern & 1967 & fusão com Western \\
Central & 1967 & fusão com Frontier \\
Cordova Airlines & 1968 fusão com Alaska \\
Northern Consolidated & 1968 fusão com Wien Air Alaska \\
Alaska Coastal & 1968 fusão com Alaska \\
Air West & 1968 & Formada pela fusão de Bonanza, Pacific e West Coast \\
Lake Central & 1968 & fusão com Allegheny \\
Trans-Caribbean & 1971 & fusão com American \\
American Flyers & 1971 & fusão com Universal \\
\hline
\end{tabular}


Tabela 1 (cont.) - Histórico de fusões de companhias aéreas de transporte de passageiros.

Fonte: Artigo "Merger Mania”, Elaboração própria.

\begin{tabular}{|c|c|c|}
\hline \multicolumn{3}{|c|}{ Ano Disposição } \\
\hline Universal & 1972 & Alguns ativos adquiridos pela Saturn \\
\hline Mohawk & 1972 & fusão com Allegheny \\
\hline Northeast & 1972 & fusão com Delta \\
\hline Caribair & 1973 & fusão com Eastern \\
\hline Saturn & 1976 & fusão com TIA \\
\hline Republic & 1979 & formada pela fusão entre North Central e Southern \\
\hline National & 1980 & fusão com Adquirida pela Pan Am \\
\hline $\begin{array}{l}\text { Hughes Air } \\
\text { West }\end{array}$ & 1980 & fusão com Republic \\
\hline Continental & 1982 & $\begin{array}{l}\text { fusão com Adquirida pela Texas Air e fusão com Texas } \\
\text { International }\end{array}$ \\
\hline Frontier & $\begin{array}{l}1985- \\
87\end{array}$ & $\begin{array}{l}\text { Adquirida pelo People Express, posteriormente comprada pela } \\
\text { Texas Air }\end{array}$ \\
\hline Key Air & 1986 & Adquirida pela Presidential \\
\hline Empire & 1986 & fusão com Piedmont \\
\hline Republic & 1986 & fusão com Northwest \\
\hline Ozark & 1986 & fusão com TWA \\
\hline New York Air & 1987 & fusão com Continental \\
\hline People Express & 1987 & fusão com Continental \\
\hline Western & 1987 & fusão com Delta \\
\hline AirCal & 1987 & fusão com American \\
\hline Jet America & 1987 & fusão com Alaska \\
\hline Florida Express & 1988 & fusão com Braniff \\
\hline PSA & 1988 & fusão com USAair \\
\hline Piedmont & 1989 & fusão com USAair \\
\hline Aspen & 1991 & fusão com Air Wincosin \\
\hline $\begin{array}{l}\text { The Trump } \\
\text { Shuttle }\end{array}$ & 1992 & $\begin{array}{l}\text { fusão com USAair assumiu as operações, fusão com US Airways } \\
\text { em } 2000\end{array}$ \\
\hline Morris Air & 1994 & fusão com Southwest \\
\hline AirTran & 1997 & Adquirida pela Valu Jet \\
\hline Carnival & 1997 & Adquirida pela Pan Am \\
\hline Reno air & 1999 & fusão com American \\
\hline $\begin{array}{l}\text { Business } \\
\text { Express }\end{array}$ & 2000 & fusão com American Eagle \\
\hline TWA & 2001 & fusão com American \\
\hline America West & 2005 & fusão com US Airways \\
\hline
\end{tabular}


Tabela 2 - Histórico de fusões de companhias aéreas de transporte de carga.

Fonte: Artigo “Merger Mania”, Elaboração própria.

\begin{tabular}{lll}
\hline Companhia & Ano & Disposição \\
\hline Seaboard World & 1980 & Fusão com Flying Tiger Line \\
Flying Tiger Line & 1989 & Fusão com Federal Express \\
Purolator Courier & 1987 & Fusão com Emery \\
Emery Worldwide & 1989 & Fusão com CF Air Freight \\
\hline
\end{tabular}

\section{Revisão de Literatura}

Se comparada com os trabalhos acerca dos efeitos dos acordos codeshare e alianças, as publicações sobre efeitos das fusões entre companhias aéreas ainda são ínfimas. Além disso, a literatura não converge, pois a análise de preços e frequência pós-fusão depende do tipo da companhia aérea em análise, se pertenciam ou não a mesma aliança, dos países de origem das companhias dentre outros fatores. Por exemplo, Brueckner e Spiller (1991) mostram que uma fusão pode levar a um aumento no bem-estar social em casos onde as firmas são "network carriers" e a demanda é alta. No entanto, Brueckner Pels (2005) mostram que, se duas companhias aéreas "network" de diferentes alianças se fundem os efeitos não competitivos prevalecem. O aumento do poder de mercado das companhias fundidas e do declínio na competição entre alianças resulta num aumento do preço e, portanto, a fusão proporciona uma redução no bem-estar social.

A tabela 3 apresenta de forma resumida os principais resultados de análises empíricas de fusões no mercado de aviação comercial em termos de seus efeitos sobre os preços dos voos e frequências. 
Tabela 2 - Resultados de fusões de companhias aéreas. Fonte: Fageda et al. (2011), Elaboração própria

\begin{tabular}{|c|c|c|c|c|c|}
\hline Autor & Ano & Companhia Aérea & País & $\begin{array}{c}\text { Efeito nos } \\
\text { preços }\end{array}$ & $\begin{array}{l}\text { Efeito nas } \\
\text { frequências }\end{array}$ \\
\hline Borenstein (1990) & 1986 & Northwest e Republic & EUA & $6 \% / 22,5 \%$ & $-28,3 \% / 53 \%$ \\
\hline Borenstein (1990) & 1986 & Trans World e Ozark & EUA & $0 \% /-12,3 \%$ & $-25,5 \% / 18,2 \%$ \\
\hline Werden et al. (1991) & 1986 & Northwest e Republic & EUA & $5,60 \%$ & $-23,70 \%$ \\
\hline Werden et al. (1991) & 1986 & Trans World e & EUA & $1,50 \%$ & $-16,20 \%$ \\
\hline Kim and Signal (1993) & $1985-1988$ & Média das fusões & EUA & $10 \%$ & \\
\hline Morrison(1996) & 1986 & Northwest e Republic & EUA & $2,50 \%$ & \\
\hline Morrison(1996) & 1986 & Trans World e Ozark & EUA & $-15,30 \%$ & \\
\hline Morrison(1996) & 1987 & Usa Air e Piedmont & EUA & $22,80 \%$ & \\
\hline Veldhius(2005) & 2004 & KLM e Air France & Europa & $0,30 \%$ & \\
\hline Peters (2006) & 1986 & Northwest e Republic & EUA & $7,20 \%$ & \\
\hline Peters (2006) & 1986 & Trans World e Ozark & EUA & $16 \%$ & \\
\hline Peters (2006) & 1986 & $\begin{array}{l}\text { Continental e People } \\
\text { Express }\end{array}$ & EUA & $29,40 \%$ & \\
\hline Peters (2006) & 1986 & Delta e Western & EUA & $11,80 \%$ & \\
\hline Peters (2006) & 1987 & Usa Air e Piedmont & EUA & $20,30 \%$ & \\
\hline Dobson e Piga (2009) & 2003 & Easyjet e Go Fly & EUA & $-12 /-27$ & \\
\hline Dobson e Piga (2009) & 2003 & $\begin{array}{l}\text { Ryanair e Buzz } \\
\text { China Southern Airlines }\end{array}$ & EUA & $-29 / 14$ & \\
\hline Zhang and Round(2009) & 2002 & $\begin{array}{lr}\text { e China } & \text { Northern } \\
\text { Airlines } & \text { Xinjian } \\
\text { Airlines, } & \\
\text { China Eastern } & \text { Airlines e }\end{array}$ & China & $-3 \%$ & \\
\hline Zhang and Round(2009) & 2002 & $\begin{array}{ll}\text { China Yunnan, China } \\
\text { Airlines e } & \text { China } \\
\text { Northwest Airlines }\end{array}$ & China & $-4 \%$ & \\
\hline Gaggero e Piga(2010) & 2007 & Era lingus e Ryanair & EUA & $7 \% / 8 \%$ & \\
\hline
\end{tabular}

O primeiro elemento a se destacar na tabela acima é o predomínio de estudos realizados para o mercado dos EUA. Poucos estudos examinam fusões fora dos Estados Unidos, provavelmente por causa da falta de dados. O "Department of Transportation, DOT" mantém uma coleta de amostra de dados de todos os bilhetes de passagem movimentados, isto viabiliza pesquisas científicas na área de Transporte e ajuda explicar este predomínio de produção científica. Segundo, a grande maioria dos estudos analisou fusões envolvendo companhias "network", enquanto apenas dois autores examinam companhias low-cost: Dobson e Piga (2009) e Gaggero e Piga (2010). Além disso, o trabalho de Gaggero et al 
(2010) é apenas uma simulação, pois a Comissão Européia, órgão similar ao CADE no Brasil, não autorizou esta operação.

Se nos concentrarmos no mercado dos EUA os resultados quase sempre nos mostram aumentos de preços e uma redução em freqüências de voo. Embora haja um número de casos em que as fusões realmente aumentem a frequência de voo, é mais comum que as frequiências diminuam. No entanto, é difícil tirar qualquer conclusão geral a respeito das freqüências, já que poucos estudos examinaram o efeito de fusões concentrando-se no mercado dos EUA.

Ao examinar o impacto sobre as tarifas, na fusão entre a "Northwest Airlines" e "Republic", é possível identificar aumentos que variam de $6 \%$, quando outras companhias aéreas estavam competindo nas rotas afetadas, para 22,5\%, quando a nova companhia aérea monopolizava a rota, segundo Borenstein (1990). Para essa mesma fusão, Werden et al. (1991) também encontraram aumentos significativos dos preços da ordem de 5,6\%, assim como Peters (2006) que relataram um aumento de 7,2\%. No entanto, o efeito foi bem mais modesto na análise realizada por Morrison (1996), não se elevando acima de 2,5\%. Seja qual for o caso, a literatura sugere que a fusão resultou em um aumento de preço.

Para a fusão entre Trans World Airlines e Ozark, o resultado não é unânime. Enquanto Borenstein (1990) e Morrison (1996) encontraram que o preço pode declinar 12,3\% e 15,3\%, respectivamente, Werden et al. (1991) e Peters (2006) relataram aumentos de preços de 1,5\% e $16 \%$. Apesar da diversidade de resultados obtidos para essa fusão, os resultados de Kim e Signal (1993) e Peters (2006) sugerem que, em geral, as fusões entre companhias tradicionais nos Estados Unidos levam a aumentos significativos dos preços.

Fora dos Estados Unidos, os resultados têm sido bastante diferentes. Fusões concluídas na China em 2002 levaram a reduções de tarifa entre 3 e 4\% segundo apontamentos de Zhang et al (2009), enquanto na Europa significativas quedas nos preços também foram encontrados, com exceção da proposta de fusão entre a Ryanair e Aer Lingus, onde Gaggero et al (2010) ressaltam que esta fusão acarretaria num aumento das tarifas. Talvez por este fato, a comissão Européia mantenha-se contrária a esta operação. Da mesma forma, Veldhius (2005) relata que a fusão entre a KLM e a Air France provavelmente não leva a aumentos significativos dos preços em rotas ligando o Noroeste da Europa a Cingapura. 
Ao examinar a literatura sobre fusões entre companhias aéreas low-cost, a primeira percepção que se tem é a escassez de trabalhos nesta área e os resultados não são convergentes. Dobson e Piga (2009) analisaram as fusões realizadas entre Ryanair e Buzz, e entre EasyJet e Go Fly, No primeiro caso, eles relatam que a fusão levou a uma mudança na estrutura de preço da Buzz, uma vez que esta foi adaptada para o sistema oferecido pela Ryanair. Enquanto que antes da fusão da Buzz eram oferecidas tarifas semelhantes em todas as datas antes do voo, no cenário pós-fusão a empresa reduziu significativamente o preço dos bilhetes comprados mais de dez dias de antecedência e aumentou significativamente o preço das tarifas compradas um dia antes da decolagem programada. Assim, a fusão resultou em preços mais baixos para os passageiros que adquirirem seus bilhetes com antecedência, mas revelou-se prejudicial para aqueles comprá-los apenas alguns dias antes. No caso da fusão entre Go Fly e EasyJet, os autores relataram uma redução significativa nas tarifas, independentemente do momento em que os bilhetes foram adquiridos.

Gaggero e Piga (2010) avaliaram os possíveis efeitos de uma fusão no mercado aéreo em que companhias aéreas low-cost operam. Este estudo mediu o nível de competição entre Aer Lingus e Ryanair e as mudanças de preços se a fusão tivesse ido à frente. Os autores relatam que as tarifas da Aer Lingus estão entre 7 e $8 \%$ menores, como resultado da concorrência com a Ryanair, e por isso as tarifas pós fusão destas duas companhias nessas rotas aumentariam neste percentual.

Até aqui, evidenciamos que a grande maioria da literatura científica têm analisado os efeitos das fusões no mercado aéreo, com ênfase geralmente nas "network carriers". Alternativamente, Dobson e Piga (2009) analisaram as fusões entre as companhias aéreas low-cost EasyJet e Go Fly, por um lado, e Ryanair e Buzz, por outro. Fageda et al (2011) extendem a literatura atual, analisando os efeitos de uma fusão entre uma companhia network e duas low-cost, a saber: Iberia - Clickair e Vueling, respectivamente. Eles baseiam-se em dados de voos domésticos do mercado aéreo espanhol para o período 2001-2010. Fageda et al fornecem evidências empíricas dos diferentes efeitos de uma fusão para diferentes tipos de companhias aéreas sob dois cenários: avaliou-se o impacto da fusão em rotas onde as duas companhias aéreas low-cost eram concorrentes previamente e, por outro lado, analisou-se os efeitos da fusão sobre rotas em que a Iberia e uma das duas low-cost estavam competindo. 
Os resultados de Fageda e Perdiguero (2011) são relevantes para determinar os efeitos de futuras fusões, desde que envolvam diferentes tipos de companhias aéreas e vale ressaltar que em sua abordagem, eles examinaram os efeitos tanto sobre os preços como sobre as freqüências da fusão. Esta consideração de freqüências é menos comum do que a análise dos preços pós-fusão. A análise de ambos os aspectos nos permite obter uma arcabouço completo do impacto da fusão no bem-estar do consumidor.

Convenientemente para a análise dos autores, a fusão foi concluída em um momento em que a Ryanair aumentou substancialmente suas operações no mercado doméstico espanhol. Desta forma, foi possível medir como a entrada de uma das principais companhias aéreas low-cost da Europa influenciou os resultados empíricos obtidos.

Fageda et al (2011) concluem que a fusão foi prejudicial para os consumidores, mas que o impacto varia de acordo com a competição entre as companhias anteriormente em rotas específicas. A fusão resultou em uma acentuada redução na frequência de vôos, mas nenhuma alteração substancial de preços nas rotas para as quais as duas companhias low-cost, onde anteriormente concorrentes. Contraditoriamente, a fusão resultou em um aumento considerável de preço, mas sem mudanças substanciais na frequência em rotas em que a companhia network e uma das duas companhias low-cost eram previamente concorrentes. Assim, o bem-estar social dos consumidores sofreu um impacto negativo, seja pela diminuição das frequências de voo ou pelas tarifas mais elevadas. No modelo, este resultado perpetuou mesmo após considerar o efeito da entrada da Ryanair em muitas rotas.

O comportamento das companhias aéreas após a fusão pode estar relacionada com os tipos diferentes de passageiros que são os usuários predominantes das rotas afetadas. As companhias aéreas exploram as elasticidades preço e frequência inerentes aos passageiros predominantes na rota a fim de maximizar suas receitas.

Os usuários a lazer são sensíveis a preço, e menos sensíveis a tempo, estão mais presentes em rotas onde as duas companhias aéreas low-cost operavam anteriormente. Por outro lado, a proporção de passageiros a negócios, os ditos: "time sensitive", parece ser maior em rotas onde a companhia network e uma das low-cost companhias operavam anteriormente. Assim, os passageiros business são mais sensíveis a diminuições nas frequências de voo por possuir um "most departure preferred time" menos flexível. 
Portanto, a resposta eficiente por parte das companhias aéreas que participam na fusão seria reduzir frequiências de voo, mas não aumentar os preços nas rotas servidas pelos dois lowcost. A combinação alta elasticidade da demanda para os preços e a baixa elasticidade da demanda para frequiências do passageiro típico nestas rotas seria responsável pelas escolhas feitas por essas companhias na tentativa de maximizar lucros. Nas rotas servidas pela network e uma das companhias aéreas low-cost, a resposta eficiente seria aumentar os preços e manter as frequências de voo. Nessas rotas, o passageiro típico parece ter uma baixa elasticidade da demanda para preços, mas uma alta elasticidade de freqüências.

\section{Tendências}

Em tempos de crise, espera-se que o movimento de fusões e aquisições mantenha-se intenso, visto que um dos principais motivos para se fusionar sociedades é a tentativa de salvar a economia de uma empresa. Por um lado, é incerto afirmar que o número destes processos aumente, diminua ou se estagne. Considerando todo o mercado brasileiro, em 2010, foram anunciados na imprensa 391 transações de fusões e aquisições, e no mesmo período deste ano registramos 359 operações.

Por outro lado, Wood et al (2010) salientam que, o crescente número de fusões se perpetuará, em diversos casos devido a tendência que as empresas tem de seguir umas às outras, ou até mesmo preferências pessoais de membros da cúpula empresarial.

\section{Conclusões}

O presente artigo apresentou recentes estudos da literatura acerca dos efeitos das fusões para o bem-estar social dos usuários do transporte aéreo, mais especificamente, como o processo impacta no preço das passagens e nas frequências de voos. Os resultados sugerem que fusões entre companhias aéreas "legacy" nos EUA tem causado significante aumento nos preços das passagens e redução nas frequências de voo. Além disso, fusões parecem resultar em redução de preços quando companhias low-cost estão envolvidas, salvo bilhetes adquiridos com poucos dias de antecedência. Por fim, fusões entre companhias network e low-cost mostramse prejudiciais para os consumidores, mas a magnitude do impacto varia de acordo com a competição entre as companhias anteriormente em rotas específicas. 
O transporte aéreo passou por um intenso processo de liberalização nas últimas décadas, como um legado, tem-se notado um aumento exacerbante do número de companhias aéreas operando em todo o mundo. Neste contexto, uma das preocupações do período atual tem sido a tendência do aumento do número de fusões e acordos codeshare entre companhias aéreas. Se por um lado, esta mudança organizacional pode garantir a sobrevivência das empresas operantes num mercado complexo, e servir como barreira às novas entrantes, por outro lado, fusões podem ser prejudiciais ao bem estar dos passageiros ao diminuir, em certos casos, a concorrência nos mercados e acarretar em tarifas onerosas e diminuição do número de frequências.

Em suma, as diferenças no impacto da fusão são substanciais, dependendo do tipo de companhia aérea envolvidas, localização geográfica e competição nos mercados e principalmente o perfil do passageiro. As companhias aéreas exploram as elasticidades preço e frequência inerentes aos passageiros predominantes na rota a fim de maximizar suas receitas: aumentar o preço das passagens em rotas onde há predominância de passageiros business, e diminuir a frequência de serviço em rotas onde há predominância de passageiros a lazer. Qualquer avaliação dos efeitos de uma fusão precisa levar em conta a percepção de que os produtos de uma "network carrier" e uma low-cost, são distintos.

\section{Referências}

Costa, W. C. (2010) Alianças Domésticas entre Companhias Aéreas e o Impacto no Comportamento dos Passageiros. Journal of Transport Literature, vol. 4, n. 1, pp. 96-113.

Dobson, P., and Piga, C. (2009) Mergers and business model assimilation: Evidence from low-cost airline takeovers. REPEC Discussion Paper Series, No.2009-2.

Fageda, X., and Perdiguero, J. (2011) An empirical analysis of a merger between a network and lowcost airlines, Working Paper.

Hamlin, G. W, (2007) Merger Mania, disponível em http://www.aca-assoc.com.

Lovadine, D. (2007) Investigação Antitruste de uma Aliança entre Operadoras de Transporte. Journal of Transport Literature, vol. 1, n. 2, pp. 7-21.

Lovadine, D. (2009) Análise Econométrica Estrutural da Conduta Competitiva: Estudo de Caso do Transporte Aéreo Pós-Liberalização Journal of Transport Literature, vol. 3, n. 1, pp. 7-39.

Vassallo, M. D. (2010) Simulação de Fusão com Variações de Qualidade no Produto das Firmas: Aplicação para o Caso do Code-Share Varig-TAM. Journal of Transport Literature, vol. 4, n. 2 , pp. 50-100.

Wood, T. J.,Vasconcelos F. C., Caldas M. P. Fusões e aquisições no Brasil. Revista de Administração de empresas RAE, vol. 43, pp. 41-45. 